\title{
CMS Payment Policy, E\&M Guideline Reform, and the Prospect of Electronic Health Record Optimization
}

\author{
Peter Basch ${ }^{1}$ Jeffery R. L. Smith ${ }^{2}$ \\ ${ }^{1}$ MedStar Health, Washington, District of Columbia, United States \\ ${ }^{2}$ American Medical Informatics Association, Bethesda, Maryland, \\ United States
}

Appl Clin Inform 2018;9:914-918.

The potential for the widespread use of electronic health records (EHRs) to improve the quality and safety of health care was a key theme of the Institute of Medicine's seminal report of 2001, "Crossing the Quality Chasm."1 EHRs were envisioned as infrastructure to a better systems-level approach to health care delivery, leveraging information technology (IT) functionality, such as point-of-care order entry and clinical decision support. This groundbreaking report further anticipated two levers that would make the capabilities within EHRs more likely to be consistently used for the purposes of care improvement-payment policy and efficiency.

It was understood that clinician payment would need to move from just paying for procedures and services, to also paying for the enhanced use of health information to improve outcomes. ${ }^{2}$ And it was assumed that operational efficiency, an intrinsic capability of mature IT seen in all other sectors, would also be seen in health care. The idea was that if physicians and other clinicians could be induced or incented to purchase and use EHRs, everyday time-consuming "paperwork" burdens would greatly diminish (or disappear!); and supported by payment policy, clinicians would make use of this new found time to focus on improving quality and safety, and perhaps even doing their part to make care more affordable.

Fast forward to 2018, and at least part of that vision is bearing fruit. While the evidence is mixed, there exists a reasonable argument for thoughtful use of EHRs resulting in better and safer care. ${ }^{3}$ However, there is scant evidence that use of EHRs has made health care delivery more efficient; to the contrary-the pre-EHR complaint of "I spend more time on paperwork than I do with patients" has been replaced with an even louder cry of "I spend more time with my EHRs than I do with patients." 4 In fact, physicians now perceive EHRs as the cause of-not a solution to-inefficiency, and the primary cause of physician burnout. ${ }^{5-7}$

received

August 28, 2018

accepted after revision

October 28, 2018

Address for correspondence Jeffery R. L. Smith, MPP, American Medical Informatics Association, 4720 Montgomery Ln. 500, Bethesda, MD 20814, United States (e-mail: jsmith@amia.org).

What happened? Implementation of IT in fields other than health care have shown (after a period of training and workflow adjustment) efficiency gains for technology endusers. ${ }^{8}$ Similar efficiency gains were assumed after the implementation of an EHR. However, persistent inefficiency in health care operations continues despite EHR implementation due to friction from two preexisting conditions: administrative or "paperwork" burdens-such as formulary adherence and prior authorizations-and the regulatory burden of documentation for payment purposes (the Evaluation and Management Documentation Guidelines [E\&M Guidelines]). Two new areas of burden further exacerbate health care inefficiency, including regulatory burden associated with specific documentation for incentive and/or quality programs, and what can be called "EHR burden"burden resulting from poor design and usability, suboptimal implementation, and inadequate training.

The proposed 2019 Medicare Physician Fee Schedule ${ }^{9}$ (PFS) represents the first time the Trump administration has sought to imprint its deregulatory ethos toward Centers for Medicare and Medicaid Services (CMS) payment policy at scale; with leadership at the Department of Health and Human Services (HHS) signaling a continuation of value-based care and a refocusing on both quality "measures that matter,"10 and the placing of "patients over paperwork,"11 as top priorities. Key among proposed changes in the PFS is a streamlining of the existing E\&M Guidelines.

This commentary will explore how a reduction in regulatory documentation burden, through E\&M Guideline reform, could favorably impact clinicians' use and interaction with EHRs, and in turn, improve the doctor-patient relationship. This commentary will not address one of the more controversial components of this proposed rule-that of creating leveling payment for outpatient office visits.

(c) 2018 Georg Thieme Verlag KG Stuttgart · New York
DOI https://doi.org/

10.1055/s-0038-1676337. ISSN 1869-0327. 


\section{Why E\&M Guideline Reform is Necessary for EHRs to Mature and Be More Usable?}

Prior to 1995 , clinician notes were expected to portray an accurate reflection of what occurred during a patient encounter, with a reasonable expectation of an appropriate history, physical exam, and assessment and plan. Clinicians essentially attested to the level of service for which they were billing with the choice of a billing code, and documentation was determined by the clinician's training and sense of professionalism. A clinician could create a long note for a less intensive visit, or a brief note for a more intensive visit. But in general, notes were brief and to the point. That all changed in 1995.

The first set of E\&M Guidelines were created as a response to what the Health Care Financing Administration (the predecessor to CMS) believed to be "code creep"; clinicians increasing their visit coding level not out of appropriateness, but rather because they could. The intent of E\&M Guidelines was to create a definition of each visit level determined not by whim, but by objective and auditable standards. An update to E\&M Guidelines, issued in 1997, attempted to make the guidelines clearer in the sections of history and physical exam, and to create more options for detailed specialty physical exams.

The response from the house of medicine was uniformly negative. By 2001, the American Medical Association (AMA) and 38 other medical professional societies labeled the guidelines as burdensome, confusing, and flawed, calling them "the single largest paperwork burden imposed by the Medicare program," and asked the CMS to reevaluate their need and value. ${ }^{12}$ HHS responded by convening an Advisory Committee on Regulatory Reform, which recommended in August of 2002 to "dump" the guidelines. ${ }^{13}$ And shortly thereafter, the Medicare Modernization Act of 2003 called for CMS to conduct pilots of new approaches to pay for physician services, without using the E\&M documentation guidelines. ${ }^{14}$ For a variety of reasons, no such pilots ever occurred.

The response from many practicing clinicians (still using paper records) was to shift from terse and meaningful handwritten or dictated notes, to the use of preprinted templatespermitting an E\&M-compliant note to be created with a combination of checkboxes and scratch outs; or a hybrid note which combined a few sentences of narrative text with preprinted forms. Physicians complained that they were spending "more time on paperwork" than they were with patients.

The E\&M Guidelines were seen by practicing clinicians as a distraction and another nonclinical encroachment on the little time they had with each patient. ${ }^{15}$ Physicians who may have spent years learning how to write a succinct note had to unlearn those skills and begin writing far longer notes that resembled those of a second year medical student. And because failure to conform to these documentation requirements carried the threat of civil and/or criminal penalties for billing fraud-physicians took compliance with these guidelines seriously, adding even more time to what they had previously spent on medical documentation.
But a slight twist of an old adage told another story... "One doctor's pain is another's business opportunity"; as the E\&M Guidelines presented an opportunity for the then-nascent commercial EHR market. Prior to the release of the E\&M Guidelines, EHRs appealed primarily to certain academic centers and technophile early adopters. But what was difficult to do by hand (following arcane documentation rules) was easy to do with an EHR-and this led to the first business case for widespread adoption: protection against failing a coding audit, which carried the threat of fines, removal from the Medicare program, and/or imprisonment. Within a few years, the business case was expanded from protection against audit failure to possibility of financial gain. A 2005 study on small practice implementation of EHRs showed a financial return on investment that came, in part, from "right coding" many E\&M services to a higher level of service, and without fear of failing a coding audit. ${ }^{16}$ And it should not come as a surprise that E\&M Guidelines never fulfilled their original purpose, which was to control costs by dissuading "code creep." 17 Both continued unabated, and along with it came an entire industry of E\&M Guidelines courses, coaches, and auditors.

What was first perceived as a "win-win" for early adopters of EHRs (improved E\&M billing profiles supported by auditproof documentation), transformed into a nightmare as more clinicians began using EHRs. To be clear, E\&M Guidelines compelled verbose and mostly meaningless clinician notes on paper, but because they were rarely shared (and mostly illegible), their impact on burden was seen almost entirely as burden on the note writer. The introduction of EHRs made the very same notes legible and easily mobilized to others-generating burden to both the note author and reader. Worse, EHR functionality and continued audit fears led to widespread overdocumentation of unnecessary and redundant information as the new normal. Clinician progress notes that were previously a few lines or paragraphs transformed into multipage narrative equivalents of "Where's Waldo" puzzles.

Indeed, the EHR era has compounded clinician burden by several orders of magnitude, while worsening the patientdoctor relationship.

\section{How Will CMS Changes Impact EHR Vendors, Clinicians, and Patients?}

Many in the informatics field have long understood that improving EHR usability takes more than improving technology. In fact, the 2011 AMIA Policy Meeting focused on the future of clinical data capture and documentation, concluding that "there is a need to transform the way we capture and document clinical care," but that reimbursement policies provide "little incentive to explore alternative data capture or documentation practices." ${ }^{18}$ A 2015 AMA-American College of Physicians-Electronic Health Record Association Usability Summit concluded further that EHRs could not fundamentally become more usable as long as the existing E\&M documentation guidelines were in place. ${ }^{19}$ While the PFS proposal would not eliminate the existing E\&M 
Guidelines, it provides two compelling alternatives that could very well provide impetus for widespread and dramatic changes to clinical note-taking and EHR usability.

CMS is proposing to undo much of the burden of documentation so that clinicians could return to documenting what is important to the clinician-patient encounter, and thus focus more of their time on patients and improving the delivery of patient care. Their proposal is to permit documentation for any outpatient physician/provider level E\&M service to either comply with the near lowest level of the existing guidelines (level 2 requirements of existing E\&M Guidelines); document on the basis of medical decision making, or MDM (the near lowest level of only one component of the existing guidelines); or documentation on the basis of time (and thus with no remnant of documentation guidelines).

To better illustrate what is being proposed, there are three areas of documentation for a medical note: history, physical exam, and MDM. ${ }^{20}$ For an established patient, the E\&M Guidelines for level 2 (what is being proposed by CMS) only require adherence to two of the three areas of documentation. What might this look like? What is shown below is only an example of what might suffice for regulatory purposes-it is presumed that the note will also contain what is professionally appropriate.

CC: Follow-up for well-controlled diabetes, hypertension, and hyperlipidemia.

HPI: The patient is doing well, and is without new complaints.

PE: BP 120/70, P 76.

Assessment and Plan (MDM): The patient is here for follow-up of well-controlled diabetes, hypertension, and hyperlipidemia.

And to restate what has already been described-for regulatory purposes the skeleton of the note shown above is even more than would be required for an established patient-where only two sections of the note would be required. E\&M University describes adherence to the guidelines for straightforward MDM as nearly impossible not to quality for it. $^{21}$

While this effort does not remove the E\&M Guidelines, it does propose a real simplification, and that coupled with the option of expanded opportunities to use time-based billing could be a substantial step forward. ${ }^{22}$ These changes could result in clinicians spending less time with each encounter, backfilling and/or overdocumenting their clinical notes, and using this new found time to either: spend more time with each patient on what is actually important; see more patients during the day; or perhaps just avoid spending every night catching up on documentation and other "EHR work." These new options to document should reduce "screen-stare" by lessening or removing the need to capture bullet-pointed and structured data. The impact on patient engagement and clinician burnout should be decidedly positive.

The potential impact on health IT may be profound. As mentioned earlier in this commentary, the primary business driver for EHRs since the mid-1990s has been to support coding for billing purposes. Leveraging the EHR such that it contained advanced visualizations of prior information and clinical notes was never a top priority, because E\&M Guideline-derived information was too bloated to be useful. This could all change, and for the better.

\section{Will Documentation Burden Decrease as a Result of This Proposal?}

That remains to be seen-as first the proposed physician fee schedule has to be finalized; and it is unlikely to be made final without revision, as there has been near universal opposition, as noted earlier in this commentary, to their proposal to link a decrease in regulatory documentation to a level payment for office visits. But even if CMS finds a way to introduce a deregulatory pathway for documentation with the 2019 Medicare PFS, its impact is likely to be less than anticipated-as this rule only is intended to address documentation for outpatient E\&M services covered by Medicare. Commensurate changes will be necessary for inpatient and emergency department services, as well as by private payers to compel widespread changes in EHRs. And finally, reducing regulatory documentation burden can only set the stage for an eventual reduction in EHR burden-it does not guarantee it will occur.

The path for clinicians may be even more challenging. Clinicians trained after 1995, when the primary arbiters of documentation shifted from clinical mentors to coders, auditors, and compliance officers, may not know any better. Changes to E\&M Guidelines should be viewed as an opportunity to review the fundamentals of clinical documentation and a means to improve documentation efficiency with EHRs. Medical professional, specialty societies, and medical educators will need to step up to train and/or retrain clinicians in documenting for twenty-first century health care. ${ }^{23}$

\section{A Reasonable Path Forward}

Clearly, widespread implementation of these policies will be difficult and time-consuming. The complex and complicated set of challenges related to clinical burden did not appear overnight, neither will remedies to address these challenges. CMS has instituted a process to improve clinician satisfaction and restore the patient-doctor relationship. The expanded documentation options proposed by CMS are a step in the right direction. Given the height of regulatory layers and the amount of money involved in our health care delivery system, none other than a stepwise approach would be prudent.

\section{Multiple Choice Questions}

1. Evaluation and Management Documentation Guidelines (E\&M Guidelines) are relevant to this ubiquitous component of electronic health records (EHRs):
a. Electronic ordering.
b. Care plan creation.
c. Clinical documentation.
d. Clinical decision support. 
Correct Answer: The correct answer is option c, clinical documentation. The Evaluation and Management Documentation Guidelines are used by both public and private payers to determine the appropriate level of service (and thus payment) for most nonprocedural (evaluation and management) services, such as office visits, emergency department visits, and hospital visits. Documentation guidelines specify what documentation is minimally required in the domains of history, physical exam, and medical decision making to justify billing at a particular level of service.

2. E\&M Guidelines are believed to make all EHRs less usable because they:

a. Have led to unnecessarily lengthy clinical notes ("note bloat").

b. Mandate a complete review of systems be documented for all encounters.

c. Force clinical documentation to be templated.

d. Clutter documentation windows with Guideline rules and explanations.

Correct Answer: The correct answer is option a, have led to unnecessarily lengthy clinical notes ("note bloat"). The number of systems included in a review of systems differs by coding level. All encounters do NOT require a complete review of systems. Many clinicians, either because they cannot remember exactly what needs to be documented for any given level of service, or due to fear of failing an E\&M coding audit, overdocument in many clinical notes. Some EHRs, particularly those developed in the 1990s, were developed as a response to the issuance of the E\&M Documentation Guidelines. These EHRs intentionally built their documentation modules as templates-such that both clinicians and auditors could easily see and count the number of necessary elements in each note. Templated documentation still exists, but is not a common feature of all EHRs. Some EHRs responded to the E\&M Documentation Guidelines not with templates, but with embedded guideline advice, reminding clinicians of what to include for any level of service in their documentation. Other EHRs built coding software that would attempt to count elements of particular components of documentation. This is also not a common feature of all EHRs. What is common is the existence of unnecessarily lengthy clinical notes-taking extra time for both the note author, and note reader.

Protection of Human and Animal Subjects

The authors certify that no human subjects were involved in development of this material.

\section{Conflict of Interest}

The authors certify that they have no affiliations with or involvement in any organization or entity with any financial interest (such as honoraria; educational grants; participation in speakers' bureaus; membership, employment, consultancies, stock ownership, or other equity interest; and expert testimony or patent-licensing arrangements), or nonfinancial interest (such as personal or professional relationships, affiliations, knowledge, or beliefs) in the subject matter or materials discussed in this manuscript. Smith acknowledges employment with the American Medical Informatics Association.

\section{References}

1 Committee on Quality Health Care in America, Institute of Medicine. Crossing the Quality Chasm: A New Health System for the 21st Century. Washington, DC: National Academy Press; 2001

2 Basch P. Electronic health records and the national health information network: affordable, adoptable, and ready for prime time? Ann Intern Med 2005;143(03):227-228

3 Kern LM, Barrón Y, Dhopeshwarkar RV, Edwards A, Kaushal R; HITEC Investigators. Electronic health records and ambulatory quality of care. J Gen Intern Med 2013;28(04):496-503

4 Sinsky C, Colligan L, Li L, et al. Allocation of physician time in ambulatory practice: a time and motion study in 4 specialties. Ann Intern Med 2016;165(11):753-760

5 Friedberg MW, Chen PG, Van Busum KR, et al. Research Report: Factors Affecting Physician Professional Satisfaction and Their Implications for Patient Care, Health Systems, and Health Policy. Santa Monica, CA: Rand Corporation; 2013

6 Downing NL, Bates DW, Longhurst CA. Physician burnout in the electronic health record era: are we ignoring the real cause? Ann Intern Med 2018;169(01):50-51

7 Khairat S, Burke G, Archambault H, Schwartz T, Larson J, Ratwani RM. Perceived burden of EHRs on physicians at different stages of their career. Appl Clin Inform 2018;9(02):336-347

8 Leonard-Barton D, Kraus W. Implementing New Technology. Harvard Business Review. November 1985. Available at: https://hbr.org/1985/ 11/implementing-new-technology. Accessed November 15, 2018

983 FR 35836. Medicare Program: Revisions to Payment Policies under the Physician Fee Schedule and Other Revisions to Part B for CY; 2019; Medicare Shared Savings Program Requirements; etc. Available at: https://www.federalregister.gov/d/2018-14985/p706. Accessed November 15, 2018

10 CMS Meaningful Measures initiative; 2017. Available at: https:// www.cms.gov/Medicare/Quality-Initiatives-Patient-AssessmentInstruments/QualityInitiativesGenInfo/CMS-Quality-Strategy.html. Accessed November 15, 2018

11 CMS Patients Over Paperwork initiative; 2018. Available at: https://www.cms.gov/About-CMS/story-page/patients-overpaperwork.html. Accessed November 15, 2018

12 Lehmann C. AMA protest convinces HHS secretary to review usefulness of E\&M guides. Psychiatric News 2001;36(16):4. Available at: https://doi.org/10.1176/pn.36.16.0004a. Accessed November 15, 2018

13 Moran M. M.D.s may soon be free of current E\&M guidelines. Psychiatric News 2002;37(15):2. Available at: https://doi.org/ 10.1176/pn.37.15.0002. Accessed November 15, 2018

14 Sec. 941 of the Medicare Modernization Act of 2003

15 Comments from Physicans Regarding AMA/HCFA. "E\&M Documentation Guidelines.” Available at: https://www.aapsonline. org/surveys/emcommen.htm. Accessed November 15, 2018

16 Miller R, West C, Martin TB, et al. The Value of Electronic Health Records in Solo Or Small Group Practices. Health Affairs. Vol. 24, No. 5. Available at: https://www.healthaffairs.org/doi/full/10.1377/hlthaff. 24.5.1127. Accessed November 15, 2018

17 Berenson RA, Basch P, Sussex A. Revisiting E\&M visit guidelines-a missing piece of payment reform. N Engl J Med 2011;364(20): 1892-1895

18 Cusack CM, Hripcsak G, Bloomrosen M, et al. The future state of clinical data capture and documentation: a report from AMIA's 2011 Policy Meeting. J Am Med Inform Assoc 2013;20(01):134-140

19 Unpublished notes and emails among the clinician participants of the AMA-ACP-EHRA Usability Summit of December 2015 
20 Level 2 Established Office Visit (99212). Available at: https:// emuniversity.com/Level2EstablishedOfficePatient.html. Accessed November 15, 2018

21 Straightforward Medical Decision-Making. Available at: https:// emuniversity.com/StraightforwardMedicalDecision-Making.html. Accessed November 15, 2018

22 Erickson SM, Rockwern B, Koltov M, McLean RM; Medical Practice and Quality Committee of the American College of Physicians.
Putting patients first by reducing administrative tasks in health care: a position paper of the American College of Physicians. Ann Intern Med 2017;166(09):659-661

23 Kuhn T, Basch P, Barr M, Yackel T; Medical Informatics Committee of the American College of Physicians. Clinical documentation in the 21st century: executive summary of a policy position paper from the American College of Physicians. Ann Intern Med 2015; 162(04):301-303 